\title{
Altered cardiac autonomic nervous function in depression
}

\author{
Yiming Wang ${ }^{1,2+}$, Xun Zhao ${ }^{1,3+}{ }^{+}$, Adrienne O'Neil ${ }^{2,4}$, Alyna Turner ${ }^{2}$, Xingde Liu ${ }^{5 *}$ and Michael Berk ${ }^{2,6,7}$
}

\begin{abstract}
Background: Depression is an independent risk factor for coronary artery disease. Autonomic instability may play a mediating or moderating role in this relationship; however this is not well understood. The objective of this study was to explore cardiac autonomic function and cardiac arrhythmia in depression, the correlation between depression severity and Heart Rate Variability (HRV) related indices, and the prevalence of arrhythmia.

Methods: Individuals ( $n=53$ ) with major depression as assessed by the Diagnostic and Statistical Manual of Mental Disorders, who had a Hamilton Rating Scale for Depression (HAMD) score $\geq 20$ and a Zung Self-Rating Depression Scale score $>53$ were compared to 53 healthy individuals, matched for age and gender. Multichannel Electrocardiograph ECG-92C data were collected over 24 hours. Long-term changes in HRV were used to assess the following vagally mediated changes in autonomic tone, expressed as time domain indices: Standard deviation of the NN intervals (SDNN), standard deviation of 5 min averaged NN intervals (SDANN), Root Mean Square of the Successive Differences (RMSSD) and percentage of NN intervals $>50$ ms different from preceding interval (pNN50). Pearson's correlations were conducted to explore the strength of the association between depression severity (using the SDS and HRV related indices, specifically SDNN and low frequency domain / high frequency domain (LF/HF)).

Results: The values of SDNN, SDANN, RMSSD, PNN50 and HF were lower in the depression group compared to the control group $(P<.05)$. The mean value of the LF in the depression group was higher than the in control group $(P<.05)$. Furthermore the ratio of LF/HF was higher among the depression group than the control group $(P<.05)$. A linear relationship was shown to exist between the severity of the depression and HRV indices. In the depression group, the prevalence of arrhythmia was significantly higher than in the control group $(P<.05)$, particularly supraventricular arrhythmias.
\end{abstract}

Conclusions: Our findings suggest that depression is accompanied by dysfunction of the cardiac autonomic nervous system, and further, that depression severity is linked to severity of this dysfunction. Individuals with depression appear to be susceptible to premature atrial and/or ventricular disease.

Keywords: Depression, Cardiac autonomic nervous system, Heart rate variability, Arrhythmia, Vagus, Cardiovascular disorders

\section{Background}

Prospective research has shown a significant relationship between depression and cardiovascular disease (CVD) [1]. In patients with coronary artery disease (CAD), cooccuring depression is associated with a range of poorer outcome measures [2-4] and a 2-4 fold increase in cardiac mortality and morbidity [5-9]. Depression has

\footnotetext{
* Correspondence: Ixdwangyiming@aliyun.com

${ }^{+}$Equal contributors

${ }^{5}$ Department of Cardiology, The Affiliated Hospital of Guiyang Medical University, Guiyang, Guizhou 550004, China

Full list of author information is available at the end of the article
}

also recently emerged as a risk factor for the onset of CVD. Individuals with a history of depression are four times more likely to have a heart attack (myocardial infarction; MI) than those without [10]. However, the underlying physiology of this relationship remains poorly understood.

Data from animal models (e.g. the chronic mild stress (CMS) model) have indicated that depression exascerbates stress reactivity, and is associated with autonomic and cardiovascular dysfunction, including reduced heart rate variability (HRV) as well as elevated heart rate (HR)

\section{Biomed Central}

(C) 2013 Wang et al.; licensee BioMed Central Ltd. This is an Open Access article distributed under the terms of the Creative Commons Attribution License (http://creativecommons.org/licenses/by/2.0), which permits unrestricted use, distribution, and reproduction in any medium, provided the original work is properly cited. 
and sympathetic cardiac tone [11-13]. Studies conducted in human populations have also reported a relationship between cardiac autonomic dysfunction and altered cardiovascular reactivity [14]. While the pathophysiologic mechanisms linking cardiovascular events to depression are unclear, there is evidence implicating lower HRV, sympathetic tone changes, arrhythmias, and changes in ventricular electrophysiological properties [15]. HRV in particular is of increasing interest, due to its clinical application [16], and has been used as an index of cardiac autonomic functioning in patients with psychiatric disorders, personality or communication disorders.

To date, studies examining HRV in patients with existing CVD have produced inconsistent results. Several studies have found that reduced HRV in patients with post-MI depression $[17,18]$ is linked to increased cardiac mortality, whereas other studies have shown no relationship between depression and low HRV in patients with stable CAD [16]. Exacerbating these inconsistencies is evidence that anxiety, which commonly co-occurs with depression, is associated with changes to HRV, and is implicated as an independent predictor of reduced parasympathetic activity in MI patients [19].

In order to elucidate the association between depression and cardiac autonomic nervous function, a comprehensive investigation of autonomic nervous system dysfunction in psychiatric populations is required. Patients with depression often suffer from symptoms of autonomic nervous system dysfunction such as palpitations occurring with a normal sinus rhythm as measured by electrocardiogram (ECG). Importantly, autonomic dysfunction and abnormal microvolt $\mathrm{T}$ wave alternans in these patients can increase the probability of sudden death [20]. We therefore sought to investigate cardiac autonomic function among individuals with and without depression, to determine the correlation between depression severity and HRV-related indices and to assess the prevalence of arrhythmia in this population.

\section{Methods}

\section{Subjects}

The depression group consisted of 53 patients (25 male, 28 female; age range $20-65$, mean $40.9 \pm 10.5$ ), who were hospitalized for major depression in the Psychiatry Department of the affiliated hospital of Guiyang Medical University between March and December, 2012.

Study participants were those with a primary diagnosis of depression. To be eligible for inclusion in the study, all subjects met diagnostic criteria for depression as assessed by the Structured Clinical Interview for Diagnostic and Statistical Manual of the American Psychiatric Association-IV-TR (DSM-IV-TR) Axis I disorders (SCID). In addition, they were assessed using the Hamilton Rating Scale for Depression (HAMD), the Zung Self- rating Depression Scale (SDS) and Hamilton Anxiety Scale (HAMA). Inclusion criteria were a $H A M D \geq 20$, a SDS $>53$ and a depression severity index range of 0.25 1.0. Exclusion criteria included a HAMA score $>14$ points (to exclude patients with primary anxiety disorders); a diagnosis of an organic depressive disorder or substance dependence; or a history of any other psychiatric illness. Patients with a current or past history of acute myocardial infarction (as assessed by ECG testing) or comorbid medical conditions including diabetes mellitus were also excluded. Anti-depressant medication usage was not an exclusion criterion, as all patients were receiving SSRIs treatment.

The control group ( $\mathrm{n}=53, \mathrm{M} 27$, F 26; age range 2063 , mean $42.1 \pm 11.61$ ) consisted of normal healthy volunteers with a HAMD score $<20$, a HAMA score $<7$, and no history of depression, anxiety or somatic disorders.

\section{Consent}

All participants gave their written informed consent to participate in the study. The procedures followed were in accordance with the revised edition of the Declaration of Helsinki of 1964 [21].

\section{Ethics}

The study was approved by the ethics Committee on the Guidelines for Experiment at Guiyang Medical University (NO 04/2012).

\section{Assessment \\ Experiment processes}

All participants were familiarized with the research protocol. Participants were precluded from taking any agents capable of influencing heart rate 48 hours prior to the experiments, such as Beta Blockers, cigarettes, alcohol, tea and coffee drinks, or from undertaking strenuous exercise within 24 hours of the assessments. Prior to the procedures, participants were asked to stay in a quiet room and rest for 15 minutes. Electrodes were attached and results analyzed by the same doctor.

\section{Dynamic electrocardiogram collection}

Twenty four hour ambulatory ECG data for all subjects was collected between 8:00 am to 8:00 am the next morning. Assessment of arrythmias was conducted by a technician who was blind to group allocation. HRV parameter values were analyzed using the ECG-92C multichannel electrocardiograph (Shanghai Photoelectric Electronic Medical Instruments Co., Ltd.). Paroxysmal tachycardia was diagnosed using repeated ECG readings $(3+)$. 


\section{The depression severity index}

The Zung Self-rating Depression Scale (SDS) is a 20-item self-report questionnaire that was used to assess depression severity. The total raw score is calculated by summing all items. The SDS depression severity index is calculated by the formula "SDS Index = Raw Score/80" [22].

\section{Time and frequency domain index absolute measures of HRV} HRV, the physiological phenomenon where the sinus cardiac rhythm varies from the mean heart rate, is measured over a 24 hour monitoring period. It is a quantitative, non-invasive and highly reproducible index of cardiac autonomic function and an important indicator of the balance of sympathetic and parasympathetic tone. It reflects the autonomic nervous control of heart function by a dynamic controlling effect [23]. Time-domain analysis indices include SDNN, SDANN, RMSSD and PNN50 by the beat-to-beat or NN intervals. The SDNN (Standard deviation of the $\mathrm{NN}$ intervals) is an overall measure of HRV size and reflects all long-term components, indicative of dysregulation of the parasympathetic and sympathetic nerve function. SDANN (standard deviation of 5 min averaged $\mathrm{NN}$ intervals) is a sensitive index of the variability of the average of 5 minute periods, and reflects circadian influences including changes in position and physical activity. Both RMSSD (Root Mean Square of the Successive Differences) and pNN50 (percentage of $\mathrm{NN}$ intervals $>50 \mathrm{~ms}$ different from preceding interval) are linked to short-term changes in HRV rather than diurnal variations, and reflect vagally mediated changes in autonomic tone.

Frequency domain (power spectral density) analysis indices $[24,25]$ at different amplitudes and frequencies describe the periodic oscillations in the decomposition of heart rate signals. This is assessed by the area (or power spectral density) of each component. Absolute values are expressed in $\mathrm{ms}^{2}$. Frequency domain indices include: the very low frequency band (VLF, <0.003$0.04 \mathrm{~Hz}, \mathrm{~ms}$ ); low-frequency power (LF, 0.04-0.15 Hz, $\mathrm{ms}^{2}$ ), a mixture of sympathetic and parasympathetic activity; high-frequency power ( $\mathrm{HF}, 0.15-0.4 \mathrm{~Hz}, \mathrm{~ms}^{2}$ ), describing parasympathetic tone; and the low-high frequency power ratio (LF/HF), which reflects the balance of sympathetic and parasympathetic innervations. The LF/HF ratio has been shown to be a good indicator of the sympathovagal balance of the autonomic nervous system [26]. HF and LF power were calculated using fast Fourier transformation (FFT). FFT is a rapid and simple nonparametric method, characterized by specified frequency windows for the frequency components. Because absolute values are more sensitive than normalized indices in the analysis of $24 \mathrm{~h} \mathrm{HRV}$ indices, absolute values of the direct measures were utilized and expressed in $\mathrm{ms}^{2}$ for the purpose of this study.

\section{Statistical analyses}

Key characteristics and clinical parameters were expressed as Means $( \pm$ SEM), with independent samples t-tests used to determine differences between the depression and control groups. Correlations between depression severity and the HRV indices, specifically SDNN and LF/HF, were assessed using Pearson's analysis. SDNN reflects the total variability over the period of recording, hence has been shown to be the most significant prognostic value among time domain parameters [27]. For each participant, the presence of an arrhythmia was defined as the occurrence of single or paired atrial and/or ventricular premature beats as detected on the ECG. Significant between group differences in the prevalence of arrhythmias were calculated using the Chi-square test. SPSS19.0 software was used and the level of significance was set at $P<.05$.

\section{Results}

\section{Demographic features}

The key characteristics of the sample are displayed in Table 1 . No statistically significant differences in demographic variables (age, sex, BP, smoking) were observed between the control and depression groups. Those in the depression group were slightly younger (40.9 years), had a higher mean systolic blood pressure (BP) and lower diastolic BP, and a higher rate of smoking than in the control group. Selective serotonin-reuptake inhibitors (SSRIs) were routinely used to treat to patients with major depression (dosing paroxetine $40 \mathrm{mg} / \mathrm{d}$ in 15 cases, escitalopram $20 \mathrm{mg} / \mathrm{d}$ in 18 cases, sertraline $100 \mathrm{mg} / \mathrm{d}$ in 20 cases).

\section{The time domain indices of HRV}

The comparison between the time domain indices of HRV in the two groups is illustrated in Table 2. In the depression group, the values of SDNN, SDANN, RMSSD, and PNN50 were significantly lower than that of the control group $(P<.05)$.

\section{The frequency domain indices of HRV}

The comparison between the mean frequency domain indices of HRV for the two groups is illustrated in

Table 1 Demographic data between the control and depression groups (Mean $\pm S E M, n=53$ ) unless indicated

\begin{tabular}{lll}
\hline Items & Control & Depression \\
\hline Ages (years) & $42.1 \pm 11.61$ & $40.9 \pm 10.5$ \\
(Ranges) & $20-63$ & $20-65$ \\
Gender (male/female) (n) & $27 / 26(51 \% / 49 \%)$ & $25 / 28(47 \% / 53 \%)$ \\
Resting SBP (mm Hg) & $125 \pm 20$ & $130 \pm 22$ \\
Resting DBP (mm Hg) & $74 \pm 9$ & $70 \pm 10$ \\
Smokers/non-smokers (n) & $24 / 29(45 \% / 55 \%)$ & $28 / 25(53 \% / 47 \%)$ \\
\hline
\end{tabular}

No significant differences in the demographic variables (age, sex, blood pressure, smoking) between the control and depression groups, were observed. 
Table 2 Comparison of time domain indices of HRV (Mean \pm SEM, $n=53$ )

\begin{tabular}{lll}
\hline Indexes $\left(\mathbf{m s}^{2}\right)$ & Depression & Control \\
\hline SDNN & $93.13 \pm 16.12^{* *}$ & $110.57 \pm 20.10$ \\
SDANN & $84.34 \pm 15.46^{*}$ & $91.09 \pm 12.69$ \\
RMSSD & $38.83 \pm 8.82^{*}$ & $43.64 \pm 11.01$ \\
PNN50 & $11.05 \pm 7.32^{*}$ & $14.07 \pm 6.72$ \\
\hline
\end{tabular}

Note: compared to control group, ${ }^{*} P<.05,{ }^{* *} P<.01$.

Table 3. The values of LF and LF/HF were significantly higher in the depression group than the control group $(P<.05)$, and the value of HF was significantly lower in the depression than the control group $(P<.05)$.

\section{The relationship between depression severity and HRV indices}

Associations between the SDS depression severity index and HRV related indices are illustrated in Figures 1 \& 2 . A linear relationship was evident between the depression severity index and the value of LF/HF (Figure 1). Pearson's relativity analysis showed a positive correlation between the two groups $(P<.05, \mathrm{r}=.335)$. A negative correlation was observed between the depression severity index and the value of SDNN (Figure 2; $P<.05 ; \mathrm{r}=-.298$ ), suggesting more severe depression was accompanied by a higher mean level of cardiac autonomic dysfunction.

\section{The prevalence of arrhythmia in depression}

There was a higher prevalence of arrhythmias, including single atrial premature beats, paired atrial premature beats and paired ventricular premature beats in the depressed group compared to the control group, $P<.05$. (Figure 3).

\section{Discussion}

This study demonstrated that, when compared with a healthy control group, patients hospitalized for clinical depression exhibit altered cardiac nervous function, evidenced by a lower high frequency domain index and a higher low frequency domain index and LF/HF ratio. Increases in the severity of depressive symptoms were shown to exacerbate these trends. Arrhythmias, particularly supraventricular arrhythmias, were over-represented in depressed patients when compared to those without.

Table 3 Comparison of frequency domain indices of HRV (Mean \pm SEM, $n=53$ )

\begin{tabular}{lll}
\hline Indexes $\left(\mathbf{m s}^{2}\right)$ & Depression & Control \\
\hline LF & $19.17 \pm 6.48^{*}$ & $16.41 \pm 5.15$ \\
HF & $18.64 \pm 4.34^{*}$ & $20.95 \pm 5.86$ \\
LF/HF & $1.05 \pm 0.35^{*}$ & $0.86 \pm 0.41$ \\
\hline
\end{tabular}

Note: compared to control group, ${ }^{*}<<.05$.

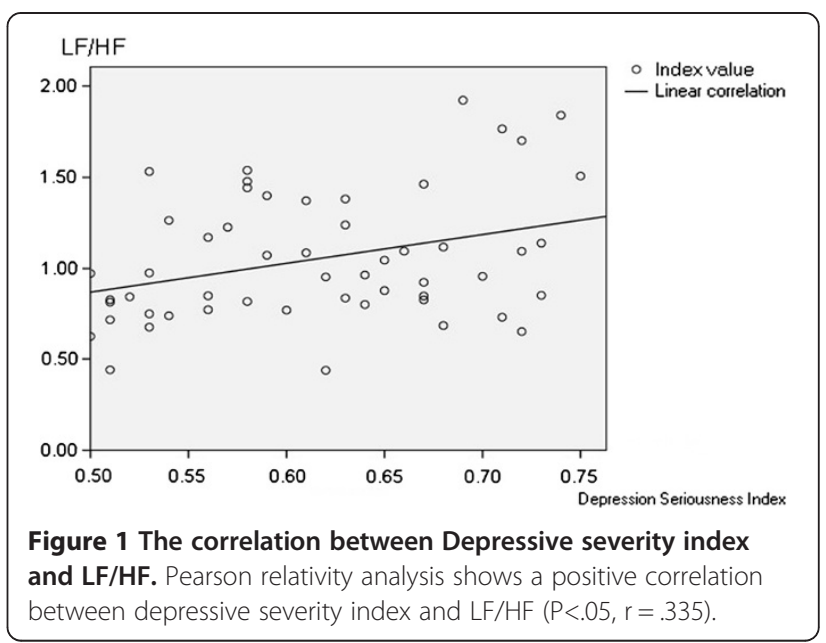

The findings from this study are consistent with the hypothesis that cardiac autonomic dysfunction is experienced by individuals with depression, especially decreased parasympathetic nerve activity (as evidenced by decreased values of the time domain indexes). This is supported by other studies of patients with depression that have applied other assessment techniques. For example, Bi et.al [28] found that patients with depression experienced autonomic nervous dysfunction using sympathetic skin response measures.

There may be a number of explanations for this finding. A mood disorder may trigger a series of adverse cardiovascular factors that evoke pathophysiological changes, which have been detailed previously [29]. These study [2-4] have demonstrated a significant relationship between depression and cardiovascular risk factors that are known to lead to adverse outcomes. Battacharyya et al. [30] hypothesized that the relationship between depression and cardiovascular risk may be explained by enhanced parasympathetic control. We suggest that the decreased $\mathrm{HF}$ and increased LF and LF/HF ratio

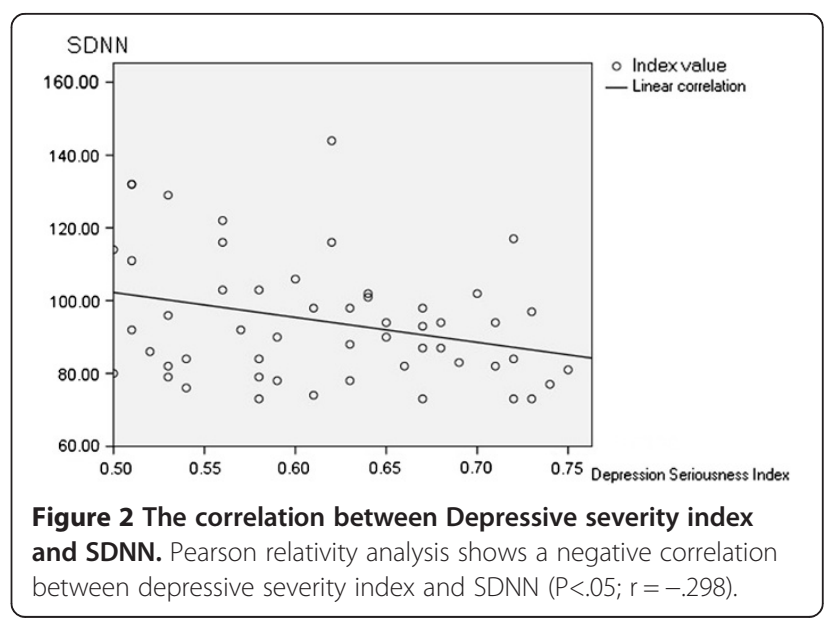




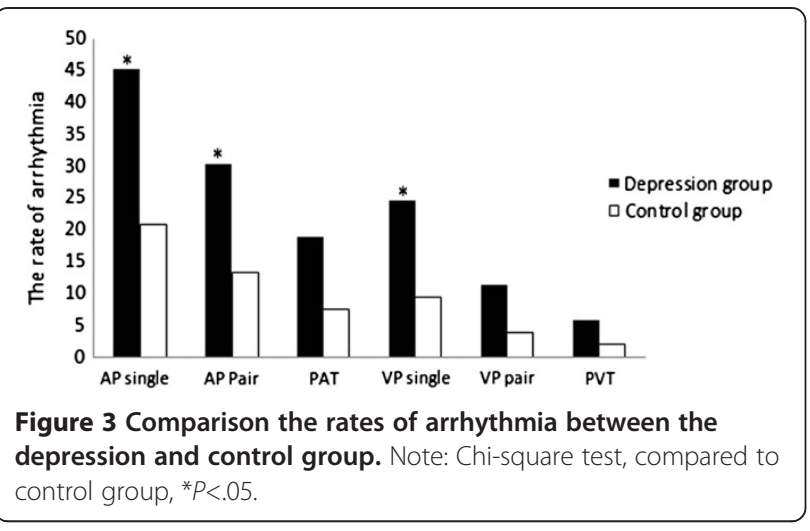

observed in our study (using frequency domain analysis) is indicative of reduced parasympathetic nerve activities and the imbalance of sympathetic and parasympathetic innervation, which may reflect dysregulation of sympathetic and parasympathetic coordination in depression. LF reflects the influence of sympathetic and parasympathetic nervous activity on heart rate fluctuation, and has relevance to vessel baroreflex effects. As such, enhanced vascular baroreceptor reflex resulting from emotional stress can induce increased LF. This is consistent with clinical manifestations in patients with depression who experience excessive tension, palpitation, chest tightness and other physical symptoms. HF may represent both vagus nerve activity and respiratory activity. However, Berger [31] found that while vagus activity was reduced in their group of depressed patients, there was no relationship between parasympathetic nervous activity and the frequency and rhythm of respiration. As there is a direct effect on HRV [32], where HRV is smaller if parasympathetic activity is lower and vice versa, we propose that parasympathetic nervous activity predominantly influences cardioregulatory function. Pathophysiological links between depression and cardiovascular system dysfunction include reduced HRV, changed sympathetic nerve activity, arrhythmia, and altered ventricular electrophysiological properties [15]. In examining HRV in depressed patients in our study, we speculated that the relative increase in sympathetic tone and corresponding reduction of parasympathetic tone may be a mechanism associated with this phenomenon and arrhythmia induction.

Our finding that a linear relationship exists between depression severity and HRV is consistent with previous research. Stein [33] found that cardiac patients with severe depression experienced significantly reduced HRV and increased mortality; a linear relationship between depression severity and level of cardiac autonomic dysfunction was observed. Agatisa [34] reported that recurrent episodes of elevated depressive symptoms were related to a greater risk of coronary and aortic atherosclerosis among those not yet diagnosed as having coronary disease. Some reports suggest the cardiac autonomic dysfunction in depressed patients may increase the risk of arrhythmia; which itself is a risk factor for coronary and other cardiovascular disorders. Indeed, we demonstrated a higher prevalence of arrhythmia in depressed patients compared with controls; a finding that may go some way to partially identifying a pathway by which depressed individuals are at increased susceptibility to CVD onset. That people with depression are more vulnerable to arrhythmias, especially supraventricular arrhythmias, such as single atrial premature beats, paired atrial premature beats and single ventricular premature beats, is in general agreement with the concept that there is a relationship between arrhythmia and emotional turbulence [35], which may be related to increased sympathetic nervous system activity [36]. Adrenergic secretion can increase the autorhythmicity of heart purkinje fibers, reducing the excited domain of the diastolic period and inducing sympathetic excitability. As a result, it may increase the probability of ventricular arrhythmia. Hyper-responsivity of sympathetic nervous activity may also constitute a risk factor for the development or progression of CVD [36].

Some of the inconsistencies in the evidence base surrounding the relationship between HRV and depression may be explained by variance in study design, assessment techniques and/or markers of autonomic functions used across studies [37]. In this study, we analyzed HRV and evaluated autonomic function through analysis of the time-domain indexes SDNN, SDANN, RMSSD and PNN50. The domain parameters of HRV used in this study are considered a reliable indicator for risk of malignant ventricular arrhythmias and sudden cardiac death when compared with ventricular late potentials, left ventricular ejection fraction, QT dispersion and the level of cardiac function $[38,39]$. Altered HRV may therefore be useful for predicting arrhythmia risk in patients with depression.

We acknowledge several limitations of this study. Ours was a sample taken from patients undergoing hospitalization and subsequent treatment for major depression. Based on the pre-determined selection criteria, they had few anxiety symptoms, a low risk of suicide, and an absence of acute myocardial infarction. While these criteria were employed in order to reduce the impact of confounding variables on our outcome of interest, this selection bias may affect the generalizability of our results. Secondly, the sample size was relatively small, limiting our ability to analyse the relationship between depression severity and HRV and perform meaningful sub-group analyses. Although it should be acknowledged that the markers of autonomic function are indirect measures, the comprehensiveness of the data including gold standard measures of depression using diagnostic 
psychiatric interviewing and HRV using domain parameters are strengths of this study. Thirdly, the cross sectional nature of the study does not allow examination of autonomic dysfunction across the disease course to determine whether remission affects this marker. Lastly, multiple comparisons were not undertaken as part of our statistical analyses; for example the effects of medication usage remain undetermined as all patients in the depressed group were receiving medication. We do acknowledge that SSRI have been shown to impact on cardiac rhythm, thus it is possible that using a sample comprising those on antidepressants may have diluted or alternatively, magnified our findings, compared with a sample from the general population, or using depressed individuals who were not on medication. Therefore, results should be interpreted with caution.

\section{Conclusions}

Our findings suggest that depression is accompanied by dysfunction of the cardiac autonomic nervous system, and further, that depression severity is linked to severity of this dysfunction. Individuals with depression appear to be susceptible to premature atrial and/or ventricular disease.

\section{Abbreviations}

CAD: Coronary artery disease; HAMD: Hamilton rating scale for depression SDS: Self-rating depression scale; CMS: Chronic mild stress; HRV: Heart rate variability; SSRIs: Selective serotonin-reuptake inhibitors; HAMA: Hamilton anxiety scale; SDS: Self-rating depression scale; SDNN: Standard deviation of the NN intervals; SDANN: Standard deviation of 5 min averaged NN intervals; RMSSD: Root mean square of the successive differences; pNN50: Percentage of $\mathrm{NN}$ intervals $>50 \mathrm{~ms}$ different from preceding interval; VLF: Very low frequency band; LF: Low-frequency power; HF: High-frequency power; LF/ HF: Ratio of low-high frequency power; FFT: Fast fourier transformation.
\end{abstract}

\section{Competing interests}

Michael Berk has received Grant/Research Support from the NIH, Cooperative Research Centre, Simons Autism Foundation, Cancer Council of Victoria, Stanley Medical Research Foundation, MBF, NHMRC, Beyond Blue, Geelong Medical Research Foundation, Bristol Myers Squibb, Eli Lilly, Glaxo SmithKline, Organon, Novartis, Mayne Pharma and Servier, has been a speaker for Astra Zeneca, Bristol Myers Squibb, Eli Lilly, Glaxo SmithKline, Janssen Cilag, Lundbeck, Meat and Livestock Board, Merck, Pfizer, Sanofi Synthelabo, Servier, Solvay and Wyeth, and served as a consultant to Astra Zeneca, Bristol Myers Squibb, Eli Lilly, Glaxo SmithKline, Janssen Cilag, Lundbeck and Servier. The other authors declare that they have no conflicts interests.

\section{Authors' contributions}

YW and $X L$ participated in the design of the study and performed the statistical analysis, MB, AO and AT participated in coordination and drafting of the manuscript. XZ carried out the studies tests. All authors participated in data interpretation, drafting of the manuscript and have read and approved the final manuscript.

\section{Acknowledgements}

This study was supported by Starting Fund of returned overseas students of Guiyang Medical University (China), 2012038. All authors had a role in either the design of study, in the collection of material, analysis and interpretation of data, in the writing of the paper; and in the decision to submit the paper for publication.

\section{Author details}

'Department of Psychiatry, The Affiliated Hospital of Guiyang Medical University, Guiyang, Guizhou 550004, China. ${ }^{2}$ IMPACT Strategic Research Centre, School of Medicine, Deakin University, Geelong 3220, Australia. ${ }^{3}$ Affiliated Hospital of Jining Medical University, Jining, Shangdong 250000, China. ${ }^{4}$ School of Public Health and Preventive Medicine, Monash University, Melbourne 3004, Australia. ${ }^{5}$ Department of Cardiology, The Affiliated Hospital of Guiyang Medical University, Guiyang, Guizhou 550004, China. ${ }^{6}$ Department of Psychiatry, Centre of Youth Mental Health, The University of Melbourne, Parkville, Melbourne, VIC 3052, Australia. ${ }^{7}$ Florey Institute of Neuroscience and Mental Health, University of Melbourne, Parkville, Melbourne, VIC 3010, Australia.

Received: 25 March 2013 Accepted: 9 July 2013

Published: 10 July 2013

\section{References}

1. Patron E, Messerotti Benvenuti S, Favretto G, Valfrè C, Bonfà C, Gasparotto R, Palomba D: Association between depression and heart rate variability in patients after cardiac surgery: a pilot study. J Psychosom Res 2012, 73:42-46

2. Tylee A, Haddad M, Barley E, Ashworth M, Brown J, Chambers J, Farmer A, Fortune Z, Lawton R, Leese M, Mann A, McCrone P, Murray J, Pariante C, Phillips R, Rose D, Rowlands G, Sabes-Figuera R, Smith A, Walters P: A pilot randomised controlled trial of personalised care for depressed patients with symptomatic coronary heart disease in South London general practices: the UPBEAT-UK RCT protocol and recruitment. BMC Psychiatry 2012, 6:12-58.

3. Charlson FJ, Stapelberg NJC, Baxter AJ, Whiteford HA: Should global burden of disease estimates include depression as a risk factor for coronary heart disease? BMC Med 2011, 9:1-6.

4. Spertus JA, McDonell M, Woodman CL, Fihn SD: Association between depression and worse disease-specific functional status in outpatients with coronary artery disease. Am Heart J 2000, 140:105-110.

5. Carney RM, Freedland KE, Miller GE, Jaffe AS: Depression as a risk factor for cardiac mortality and morbidity: a review of potential mechanisms. J Psy chosom Res 2002, 53:897-902.

6. Nicholson A, Kuper $\mathrm{H}$, Hemingway $\mathrm{H}$ : Depression as an aetiologic and prognostic factor in coronary heart disease: a meta-analysis of 6362 events among 146538 participants in 54 observational studies. Eur Heart J 2006, 27:2763-2774.

7. Barth J, Schumacher M, Herrmann-Lingen C: Depression a risk factor for mortality in patients with coronary heart disease: a meta-analysis. Psychosom Med 2004, 66:802-813.

8. Penninx BW, Beekman AT, Honig A, Deeg DJ, Schoevers RA, van Eijk JT, van Tilburg W: Depression and cardiac mortality: results from a communitybased longitudinal study. Arch Gen Psychiatry 2001, 58:221-227.

9. Whang W, Kubzansky LD, Kawachi I, Rexrode KM, Kroenke CH, Glynn RJ, Garan H, Albert CM: Depression and risk of sudden cardiac death and coronary heart disease in women: results from the nurses' health study. J Am Coll Cardiol 2009, 53:950-958.

10. Pratt LA, Ford DE, Crum RM, Armenian HK, Gallo JJ, Eaton WW: Depression, psychotropic medication, and risk of myocardial infarction: prospective data from the Baltimore ECA Follow-up. Circulation 1996, 94:3123-3129.

11. Grippo AJ, Beltz TG, Johnson AK: Behavioral and cardiovascular changes in the chronic mild stress model of depression. Physiol Behav 2003, 78:703-710.

12. Carney RM, Saunders RD, Freedland KE, Stein P, Rich MW, Jaffe AS: Association of depression with reduced heart rate variability in coronary artery disease. Am J Cardiol 1995, 76:562-564.

13. Strekalova T, Spanagel R, Bartsch D, Henn FA, Gass P: Stress-induced anhedonia in mice is associated with deficits in forced swimming and exploration. Neuropsychopharmacology 2004, 29:2007-2017.

14. Ehrenthal JC, Herrmann-Lingen C, Fey M, Schauenburg H: Altered cardiovascular adaptability in depressed patients without heart disease. World J Biol Psychiatry 2010, 11:586-593.

15. Khawaja IS, Westermeyer JJ, Gajwani P, Feinstein RE: Depression and coronary artery disease: the association, mechanisms, and therapeutic implications. Psychiatry (Edgemont) 2009, 6:38-51.

16. Gehi A, Mangano D, Pipkin S, Browner WS, Whooley MA: Depression and heart rate variability in patients with stable coronary heart disease: findings from the heart and soul study. Arch Gen Psychiat 2005, 62:661-666. 
17. Carney RM, Blumenthal JA, Stein PK, Watkins L, Catellier D, Berkman LF, Czajkowski SM, O'Connor C, Stone PH, Freedland KE: Depression, heart rate variability, and acute myocardial infarction. Circulation 2001, 104:2024-2028.

18. Rich MW, Saini JS, Kleiger RE, Carney RM, te Velde A, Freedland KE: Correlation of heart rate variability with clinical and angiographic variables and late mortality after coronary angiography. Am J Cardiol $1988,62: 714-717$.

19. Martens EJ, Nyklicek I, Szabo BM, Kupper N: Depression and anxiety as predictors of heart rate variability after myocardial infarction. Psychol Med 2008, 38:375-383.

20. Huikuri HV, Mäkikallio TM, Raatikainen MJP, Perkiömäki P, Castellanos A, Myerburg RJ: Prediction of sudden death: appraisal of the studies and methods assessing the risk of sudden arrhythmic death. Circulation 2003, 108:110-115.

21. Rickham PP: Human Experimentation. Code of ethics of the world medical association. Br Med J 1964, 2:177

22. Zhou KN, Li XM, Yan H, Dang SN, Wang DL: Effects of music therapy on depression and duration of hospital stay of breast cancer patients after radical mastectomy. Chin Med J (Engl) 2011, 124:2321-2327.

23. Rajendra AU, Paul JK, Kannathal N, Lim CM, Suri JS: Heart rate variability: a review. Med Bio Eng Comput 2006, 44:1031-1051.

24. Hemingway $H$, Shipley $M$, Brunner $E$ : Does autonomic function link social position to coronary risk. Circulation 2005, 111:3071-3077.

25. Park SB, Lee BC, Jeong KS: Standardized tests of heart rate variability for autonomic function tests in healthy Koreans. Int J Neurosci 2007, 117:1707-1717.

26. Wang HM, Huang SC: SDNN/RMSSD as a surrogate for LF/HF: a revised investigation. Model and Simul Engin 2012. Article ID 931943, htt://dx.doi. org/10.1155/2012/931943.

27. Sredniawa B, Musialik-Lydka A, Herdyńska-Was M, Pasyk S: The assessment and clinical significance of heart rate variability. Pol Merkur Lekarski 1999, 7:283-288.

28. Bi Y, Hu XY, Wang Z: Research on sympathetic skin response in patients with depression. Chin J Phys Med Rehabil 2008, 30:819-822.

29. Li XH, Zhao RT, Guo YF, Hu YM, Zhang W, Zhao M, Yao L, Yang JG: The impact of depression on heart rate variability in patients with coronary heart disease. Chin Med Hera 2010, 12:1145-1147.

30. Bhattacharyya MR, Whitehead DL, Rakhit R, Steptoe A: Depressed mood, positive affect, and heart rate variability in patients with suspected coronary artery disease. Psychosom Med 2008, 70:1020-1027.

31. Berger S, Kliem A, Yeragani V, Bär KJ: Cardio-respiratory coupling in untreated patients with major depression. J Affect Disord 2012 139:166-171.

32. Xu YG, Zhou SH, Li YG, Shen XQ, Hu XQ, Fang ZF, Chen S, Xu DM: Correlation between heart rate variability and coronary artery pathology in patients with coronary artery disease. Heart J 2007, 19:30-33.

33. Stein PK, Carney RM, Freedland KE, Skala JA, Jaffe AS, Kleiger RE, et al: Severe depression is associated with markedly reduced heart rate variability in patients with stable coronary heart disease. J Psychosom Res 2000, 48:493-500.

34. Agatisa PK, Matthews KA, Bromberger JT, Edmundowicz D, Chang YF, Sutton-Tyrrell K: Coronary and aortic calcification in women with history of major depression. Arch Intern Med 2005, 165:1229-1236.

35. Zhou J, Li XP, Zhao SP,Yuan ZY, Wu JB, Xia X, Fan M: The clinical analysis of anxiety depression in cardiovascular patients. J Med Theor \& Pra 2004, 17:15-17.

36. Rozanski A, Blumenthal JA, Kaplan J: Impact of psychological factors on the pathogenesis of cardiovascular disease and implications for therapy. Circulation 1999, 99:2192-2217.

37. Lisa M, Christian MA, Catherine M, Stoney PHD: Social support versus social evaluation: unique effects on vascular and myocardial response patterns. Psychosom Med Psychosom Med 2006, 68:914-921.
38. Rodrigues TR, Miranda RC, Lichter AP, Lobo NC, Figueroa CS, da Consolação MM: Heart rate variability in myocardial infarction with and without malignant arrhythmias: comparison with heart transplant recipients and normal subjects. Pacing Clin Electrophysiol 1996, 19:1857-1862.

39. Bigger JT, Fleiss JL, Rolnitzky LM, Steinman RC: The ability of several shortterm measures of RR variability to predict mortality after myocardial infarction. Circulation 1993, 88:927-934.

doi:10.1186/1471-244X-13-187

Cite this article as: Wang et al:: Altered cardiac autonomic nervous function in depression. BMC Psychiatry 2013 13:187.

\section{Submit your next manuscript to BioMed Central and take full advantage of:}

- Convenient online submission

- Thorough peer review

- No space constraints or color figure charges

- Immediate publication on acceptance

- Inclusion in PubMed, CAS, Scopus and Google Scholar

- Research which is freely available for redistribution

Submit your manuscript at www.biomedcentral.com/submit
C Biomed Central 